\title{
Reliability Measures for Fuel System in Diesel Engine
}

\author{
Rekha Choudhary, PhD. \\ Department of Mathematics \\ Govt. Engineering College, \\ Bharatpur, (Rajasthan), India
}

\author{
Viresh Sharma, PhD. \\ Department of Mathematics \\ N.A.S. (PG) College, Meerut \\ (U.P), India
}

\author{
Sonendra Kumar Gupta \\ Department of Mathematics \\ Oriental College of \\ Technology, Bhopal (MP), \\ India
}

\begin{abstract}
The authors have considered here helps to transfer the fuel from the fuel tank to the engine and regulate its flow into the cylinder depending on speed and local requirement. Here, a multi-component fuel system in diesel engine, comprised two subsystems $A$ and $B$ in series has been considered. In this system the fuel is injected into the cylinder using an injection pump and injectors. The block diagram depicts the fuel system in an $M$-cylinder diesel engine. The diesel fuel from the tank is pump to the fuel injection device. This device consists of small plunger pumps operating to push the fuel through the injectors (nozzles) into the cylinders one can operated one pump from the fuel injection pumps at the appropriate time i.e., towards the end of the compression stroke the fuel is pushed through the small hole in the injector and is atomized as it enters into the cylinder.
\end{abstract}

\section{Keywords}

Supplementary variable techniques, Laplace transform, Abel's Lemma, Cost function, availability function and reliability function.

\section{INTRODUCTION}

Suppose a multi-component fuel system in diesel engine, comprised two subsystems $A$ and $B$ in series has been considered. In this system the fuel is injected into the cylinder using an injection pump and injectors. The block diagram depicts the fuel system in an $M$-cylinder diesel engine. The diesel fuel from the tank is pump to the fuel injection device. This device consists of small plunger pumps operating to push the fuel through the injectors (nozzles) into the cylinders one can operated one pump from the fuel injection pumps at the appropriate time i.e., towards the end of the compression stroke the fuel is pushed through the small hole in the injector and is atomized as it enters into the cylinder.

Now $M$-cylinders is $j$-out-of-M: $D$ system) more than $\mathrm{M}-j+1$ cylinders s must fail for $A$ to fail), while sub-system $B$ may fail due to failure of anyone of its units. Also the whole system can fail due to critical human error and environmental pressure from the normal efficiency and degraded state.

The lifetime of the active units depend on each other in having simultaneous failure of all the operating units and repair times are distributed quite generally. $A$ failed unit is repaired at a single service channel. [1], [2], [3] Earlier researchers have studied the model for $j=1,2$; under the assumption that the repair of a unit is possible only when the system stops operating and the life times of the active units are mutually independent, and have studied the model with exponential failure distribution under the different repair policies. [2] The concept of human error and environmental pressure has been examined. The availability and reliability function for the system are obtained simultaneously by using supplementary variable technique.

\section{ASSUMPTIONS}

(i). Initially, all the units are operating.

(ii). Failure rates are exponential.

(iii).The system goes to complete breakdown if more than $M$ $j$ units in A are simultaneously failed or if any failure in B occurs, critical human error or under environmental disasters.

(iv). A failed unit is repaired at a single service channel.

(v). A repaired unit is put into the operation immediately.

(vi). A repaired unit is assumed to behave like new after repair.

(vii).Repair times are distributed quite generally. The system has three modes; good, degraded and failed state.

(viii).The system has three modes; good, degraded and failed state.

\section{NOTATIONS}

$\begin{array}{ll}\int & : \int_{0}^{\infty} \text { Otherwise stated } \\ M, N & : \text { Number of units in subsystem } A \text { and } B \\ \lambda / \lambda^{\prime} / \lambda^{\prime \prime} / e_{1} & : \text { Constant failure rates from state } 0 \text { to } Q \\ / e_{2} / h_{1} / h_{2} & 0 \text { to } 3 / 0 \text { to } 1 / 1 \text { to } 2 / 0 \text { to } 5 / 1 \text { to } 5 / 0 \text { to } 6 / \\ & \text { to } 6 . \\ \phi_{i}(x), S_{i}(x) & : \text { Transition repair rate of the subsystem } \\ & B / \text { pdf of repair rate } \\ \phi(r) & : \text { Transition repair rate of subsystem } A \\ & p d f \text { of repair rate }\end{array}$


$\phi_{/ /}(z) \quad:$ Transition repair rate for critical human error

$\phi_{e}(k) \quad:$ Transition repair rate for environmental Pressure

$\bar{f}(s) \quad:$ Laplace transform of $f(t)$

$P_{M . N}(t) \quad:$ The probability that at time $t$, the system is operating in the state of normal efficiency.

$P_{M-j . N}(\mathrm{y}, t) \Delta:$ The probability that at time $t$, the system is in degraded state due to the failure units of subsystem $A$ and elapsed repair time lies in the interval $(\mathrm{y}, \mathrm{y}+\Delta)$

$P_{M-j+1 . N}(r, t) \Delta:$ The probability that at time $t$, the system is in the failed state due to the failure of $M-j+1$ units of subsystem $A$. The elapsed repair time lies in the $(r, r+\Delta)$.

$P_{M . i}(x, t) \quad:$ The probability that at time $t$, the system is in failed state due to the failure of $i^{\text {th }}$ unit of subsystem $B$. The elapsed repair time lies in the interval $(x, x+\Delta)$.

$P_{M-j . i}(x, t) \Delta \quad:$ The probability that at time $t$ the system is in failed state. The elapsed repair time lies in the interval $(x, x+\Delta)$.

$P_{H}(z, t) \quad:$ The probability that at time $t$ the system is in failed state due to critical human error. The elapsed repair time lies in interval $(z, z+\Delta)$.

$P_{e}(k, t) \quad:$ The probability that at time $t$ the system is in failed state due to environmental disorders. The elapsed repair time lies in the interval $(k, k+\Delta)$.

\section{FORMULATION OF THE MATHEMATICAL MODEL}

The analysis crucially depends on the method of supplementary variables, and the supplementary variable $\times$ denotes the elapsed time that a unit has been undergoing repair. Viewing the nature of the problem, the following set of difference-differential equations is obtained as follows:

$$
\begin{aligned}
& {\left[\frac{\partial}{\partial t}+\lambda+\lambda^{\prime}+e_{1}+h_{1}\right] P_{M, N}(t)=\sum_{p=1}^{j} \int P_{M-j . N}(y, t) \phi_{p}(y) d y} \\
& +\sum_{i=1}^{M} \int P_{M, i}(x, t) \phi_{i}(x) d x \\
& +\int P_{M-j+1, N}(r, t) \phi(r) d r+\int P_{e}(k, t) \phi_{e}(k) d k+ \\
& \int P_{/ /}(z, t) \phi_{/ /}(z) d z \\
& {\left[\frac{\partial}{\partial y}+\frac{\partial}{\partial t}+\lambda+\lambda "+e_{2}+h_{2}+\phi_{p}(y)\right] P_{M-j, N}(y, t)=0 \ldots(} \\
& {\left[\frac{\partial}{\partial r}+\frac{\partial}{\partial r}+\phi(r)\right] P_{M-j+1, N}(r, t)=0} \\
& {\left[\frac{\partial}{\partial x}+\frac{\partial}{\partial t}+\phi_{i}(x)\right] P_{M, 1}(x, t)=0} \\
& {\left[\frac{\partial}{\partial x}+\frac{\partial}{\partial t}+\phi_{i}(x)\right] P_{M-j, i}(x, t)=0} \\
& {\left[\frac{\partial}{\partial k}+\frac{\partial}{\partial t}+\phi_{e}(k)\right] P_{e}(k, t)=0} \\
& {\left[\frac{\partial}{\partial z}+\frac{\partial}{\partial t}+\phi_{/ /}(z)\right] P_{/ /}(z, t)=0}
\end{aligned}
$$

These equations can be solved under following boundary and initial conditions:

\subsection{Boundary Conditions}

$$
\begin{aligned}
& P_{M-j, N}(0, t)=\lambda^{\prime} P_{M, N}(t) \sum_{i=1}^{M} \int P_{M-j, i}(x, t) \phi_{i}(x) d x \\
& \quad+\int P_{e}(k, t) \phi_{e}(k) d k+\int P_{H}(z, t) \phi_{H}(z) d z \\
& P_{M-j+1, N}(0, t)=\lambda^{\prime \prime} P_{M-j, N}(t) \\
& P_{M, t}(0, t)=\lambda P_{M, N}(t) \\
& P_{M-j, t}(0, t)=\lambda P_{M-i, N}(t) \\
& P_{e}(0, t)=e_{1} P_{M, N}(t)+e_{2} P_{M-j, N}(t) \\
& P_{H}(0, t)=h_{1} P_{M, N}(t)+h_{2} P_{M-j, N}(t)
\end{aligned}
$$

\subsection{Initial Condition}

$$
P_{k}(0)=1 \text {, as } k=M, N \text { otherwise zero }
$$




\section{CYLINDRICAL FUEL SYSTEM AND TRANSITION STATE DIAGRAM}

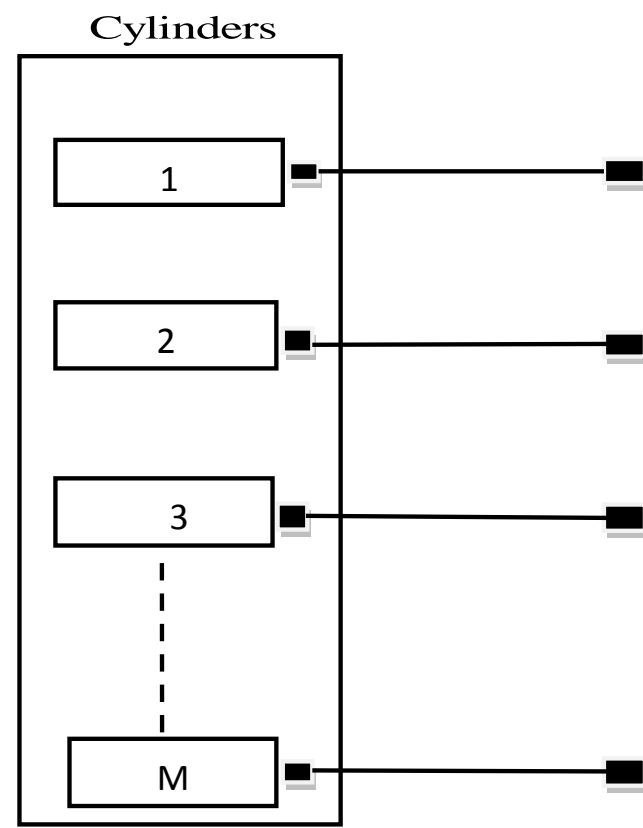

Subsytem A

Fuel System in Diesel Engine

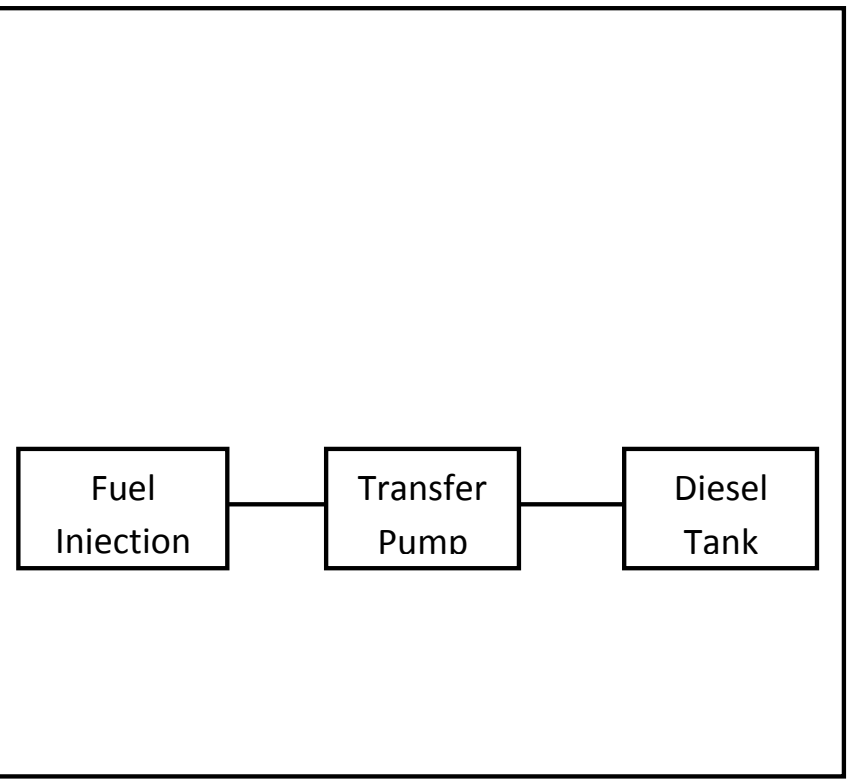

Subsytem B

Figure 1: Block Diagram of Cylindrical Fuel System






\section{SOLUTIONS OF MATHEMATICAL MODEL}

Taking Laplace transforms of the equations (1) through (13) and using initial conditions we may get:

$$
\begin{aligned}
& {\left[s+\lambda+\lambda^{\prime}+e_{1}+h_{1}\right] \bar{P}_{M, N}(s)=1+\sum_{p=1}^{j} \int \bar{P}_{M-j, N}(y, s) \phi_{p}(y) d y} \\
& +\sum_{i=1}^{M} \int \bar{P}_{M, i}(x, s) \phi_{i}(x) d x+\int \bar{P}_{M-j+1, N}(r, s) \phi(r) d r \\
& +\int \bar{P}_{e}(k, s) \phi_{e}(k) d k+\int \bar{P}_{H}(z, s) \phi_{H}(z) d z \\
& {\left[\frac{\partial}{\partial y}+s+\lambda+\lambda^{\prime}+e_{2}+h_{2}+\phi_{p}(y)\right] \bar{P}_{M-j, N}(y, s)=0} \\
& {\left[\frac{\partial}{\partial r}+s+\phi(r)\right] \bar{P}_{M-j+1, N}(r, s)=0} \\
& {\left[\frac{\partial}{\partial x}+s+\phi_{i}(x)\right] \bar{P}_{M, 1}(x, s)=0} \\
& {\left[\frac{\partial}{\partial x}+s+\phi_{i}(x)\right] \bar{P}_{M, j, i}(x, s)=0} \\
& {\left[\frac{\partial}{\partial k}+s+\phi_{e}(k)\right] \bar{P}_{e}(k, s)=0} \\
& {\left[\frac{\partial}{\partial z}+s+\phi_{H}(z)\right] \bar{P}_{H}(z, s)=0} \\
& \bar{P}_{M-j, N}(0, s)=\lambda^{\prime} \bar{P}_{M, N}(s)+\sum_{i=1}^{M} \int \bar{P}_{M-j, i, s}(x, s) \phi_{i}(x) d x \\
& +\int \bar{P}_{e}(k, s) \phi_{e}(k) d k+\int \bar{P}_{H}(z, s) \phi_{H}(z) d z \\
& \bar{P}_{M-j+1, N}(0, s)=\lambda " \bar{P}_{M-j, N}(s) \\
& \bar{P}_{M, i}(0, s)=\lambda \bar{P}_{M, N}(s) \\
& \bar{P}_{M-j, i}(0, s)=\lambda \bar{P}_{M-j, N}(s) \\
& \bar{P}_{e}(0, s)=e_{1} \bar{P}_{M, N}(s)+e_{2} \bar{P}_{M-j, N}(s) \\
& P_{H}(0, s)=h_{1} \bar{P}_{M, N}(s)+h_{2} \bar{P}_{M-j, N}(s)
\end{aligned}
$$

Integrating (18) and using (24) one obtain:

$$
\begin{aligned}
& \bar{P}_{M, j}(x, s)=\lambda \bar{P}_{M, N}(s) \exp \cdot\left[-s x-\int_{0}^{x} \phi_{i}(x) d x\right] \\
& \bar{P}_{M, i}(s)=\lambda \bar{P}_{M, N}(s) D_{\phi_{i}}(s)
\end{aligned}
$$

Integrating equation (18) by making use of (24) we get:

$$
\begin{aligned}
\bar{P}_{M-j, i}(x, s) & =\lambda \bar{P}_{M-j, N}(s) \exp \cdot\left[-s x-\int_{0}^{x} \phi_{i}(x) d x\right] \\
\bar{P}_{M-j, i}(s) & =\lambda \bar{P}_{M-j, N}(s) D_{\phi_{i}}(s)
\end{aligned}
$$

Integrating (17) using (23) one may get

$$
\bar{P}_{M-j+1, i}(r, s)=\lambda " \bar{P}_{M-j, N}(s) \exp \cdot\left[-s x-\int_{0}^{r} \phi(r) d r\right]
$$$$
\bar{P}_{M-j+1, i}(s)=\lambda " \bar{P}_{M-j, N}(s) D_{\phi}(s)
$$

Integrating (20) and using (26) one has

$\bar{P}_{e}(k, s)=\left[e_{1} \bar{P}_{M, N}(s)+e_{2} \bar{P}_{M-j . N}(s)\right] \exp .\left[-s k-\int_{0}^{k} \phi_{e}(k) d k\right]$

$$
\bar{P}_{e}(s)=\left[e_{1} \bar{P}_{M, N}(s)+e_{2} \bar{P}_{M-j . N}(s)\right] D_{\phi_{e}}(s)
$$

Integrating (21) and using (27) one may have

$$
\begin{aligned}
& \bar{P}_{H}(z, s)=\left[h_{1} \bar{P}_{M, N}(s)+h_{2} \bar{P}_{M-j . N}(s)\right] \exp \cdot\left[-s z-\int_{0}^{z} \phi_{H}(z) d z\right] \\
& \bar{P}_{H}(s)=\left[h_{1} \bar{P}_{M, N}(s)+h_{2} \bar{P}_{M-j . N}(s)\right] D_{\phi_{H}}(s)
\end{aligned}
$$

Integrating (16) by using relevant relations one may obtain: $\bar{P}_{M-j, N}(y, s)=G(s) \bar{P}_{M, N}(s)$

$$
\begin{aligned}
& G(s)=\left[\lambda^{\prime}+e_{1} \bar{S}_{\phi_{e}}(s)+h_{1} \bar{S}_{\phi_{H}}(s)\right] \\
& \quad \times D_{\phi_{r}}\left(s+\lambda^{\prime}+\lambda^{\prime \prime}+e_{2}+h_{2}-\lambda \bar{S}_{\phi_{i}}(s)-e_{2} \bar{S}_{\phi_{e}}(s)-h_{2} \bar{S}_{\phi_{H}}(s)\right)
\end{aligned}
$$

Where, $D_{k}(s)=\frac{1-\bar{S}_{k}(s)}{s}, \forall k$

Lastly, equation (15) gives by using relevant relations

$$
\bar{P}_{M, N}(s)=\frac{1}{A(s)}
$$

Where,

$$
\begin{array}{r}
A(s)=s+\lambda+\lambda^{\prime}+e_{1}\left[1-\bar{S}_{\phi_{e}}(s)\right]+h_{1}\left[1-\bar{S}_{\phi_{H}}(s)\right] \\
-\left(\lambda^{\prime}+e_{1} \bar{S}_{\phi_{e}}(s)+h_{1} \bar{S}_{\phi_{H}}(s)\right) \bar{S}_{p}(s)\left[s+\lambda^{\prime}+\lambda^{\prime \prime}+e_{2}\right. \\
\left.+h_{2}-\lambda \bar{S}_{\phi_{i}}(s)-e_{2} \bar{S}_{\phi_{e}}(s)-h_{2} \bar{S}_{\phi_{H}}(s)\right] \\
\times\left[s+\lambda^{\prime}+\lambda^{\prime \prime}+e_{2}+h_{2}-\lambda \bar{S}_{\phi_{i}}(s)-e_{2} \bar{S}_{\phi_{e}}(s)-h_{2} \bar{S}_{\phi_{H}}(s)\right] \\
{\left[e_{2} \bar{S}_{\phi_{e}}(s)+h_{2} \bar{S}_{\phi_{H}}(s)+\lambda^{\prime \prime} \bar{S}_{\phi}(s)\right] G(s)}
\end{array}
$$

Thus finally we have

$$
\begin{aligned}
& \bar{P}_{M, N}(s)=\frac{1}{A(s)} \\
& \bar{P}_{M-j, N}(s)=\frac{G(s)}{A(s)} \\
& \bar{P}_{M-j+1, N}(s)=\frac{\lambda " G(s)}{A(s)} D_{\phi}(s) \\
& \bar{P}_{M, j}(s)=\frac{\lambda}{A(s)} D_{\phi}(s) \\
& \bar{P}_{M-j, i}(s)=\frac{\lambda G(s)}{A(s)} D_{\phi}(s) \\
& \bar{P}_{e}(s)=\frac{1}{A(s)}\left[e_{1}+e_{2} G(s)\right] D_{\phi}(s) \\
& \bar{P}_{H}(s)=\frac{1}{A(s)}\left[h_{1}+h_{2} G(s)\right] D_{\phi}(s)
\end{aligned}
$$

\section{ERGODIC BEHAVIOR OF THE SYSTEM}

Using Abel's Lemma in L.T. viz.

$\lim _{s \rightarrow 0} s \bar{F}(s)=\lim _{t \rightarrow \infty} F(t)=F($ Say) Provided the limit on R.H.S. 
exists, the time independent probabilities are obtained as follows:

$$
\begin{aligned}
& P_{M, N}=\frac{1}{A^{\prime}(0)} \\
& P_{M-j, N}=\frac{G(0)}{A^{\prime}(0)} \\
& P_{M-j+1, N}=\frac{\lambda^{\prime \prime} G(0)}{A^{\prime}(0)} M_{\phi}(s) \\
& P_{M, t}=\frac{\lambda}{A^{\prime}(0)} M_{\phi} \\
& P_{M-j, t}=\frac{\lambda G(0)}{A^{\prime}(0)} M_{\phi} \\
& P_{e}=\frac{1}{A^{\prime}(s)}\left[e_{1}+e_{2} G(0)\right] M_{\phi} \\
& P_{e}=\frac{1}{A^{\prime}(0)}\left[h_{1}+h_{2} G(0)\right] M_{\phi}
\end{aligned}
$$

Where,

$$
M_{k}=-\bar{S}_{k}{ }^{\prime}(0)=\text { Mean time to repair } k^{\text {th }} \text { unit }
$$

\section{SOME PARTICULAR CASES}

When the repair follow exponential time distribution

Setting $\bar{S}_{\phi_{i}}(s)=\frac{\phi_{i}}{s+\phi_{i}}, \bar{S}_{\phi_{e}}(s)=\frac{\phi_{e}}{s+\phi_{e}}$ etc. in equation through (41) one obtains:

$$
\begin{aligned}
& \bar{P}_{M, N}(s)=\frac{1}{E(s)} \\
& \bar{P}_{M-j, N}(s)=\frac{g(s)}{E(s)} \\
& \bar{P}_{M-j+1, N}(s)=\frac{\lambda " g(s)}{E(s)} \frac{1}{s+\phi} \\
& \bar{P}_{M, i}(s)=\frac{\lambda}{E(s)} \frac{1}{s+\phi_{i}} \\
& \bar{P}_{M-j, i}(s)=\frac{\lambda g(s)}{E(s)} \frac{1}{s+\phi_{i}} \\
& \bar{P}_{e}(s)=\frac{1}{E(s)}\left[e_{1}+e_{2} g(s)\right] \frac{1}{s+\phi_{i}} \\
& \bar{P}_{H}(s)=\frac{1}{E(s)} \frac{1}{s+\phi_{H}}\left[h_{1}+h_{2} g(s)\right]
\end{aligned}
$$

Where, $\quad E(s)=[A(s)]_{\bar{S} k(s)=\frac{k}{s+k}}, g(s)=[G(s)]_{\bar{S} k(s)=\frac{k}{s+k}}$

\section{EVALUATION OF UP AND DOWN STATE PROBABILITIES}

$$
\begin{aligned}
\bar{P}_{U P}(s) & =\bar{P}_{M, N}(s)+\bar{P}_{M-j, N}(s) \\
& =\frac{1}{s+\lambda+\lambda^{\prime}+e_{1}+h_{1}}\left[1+\frac{\lambda^{\prime}}{s+\lambda^{\prime}+\lambda^{\prime \prime}+e_{2}+h_{2}}\right]
\end{aligned}
$$

Inverting this we have

$$
\begin{aligned}
P_{u p}(t)=(1+A) \exp [ & \left.-\left(\lambda+\lambda^{\prime}+e_{1}+h_{1}\right) t\right] \\
& +B \exp \left[-\left(\lambda^{\prime}+\lambda^{\prime \prime}+e_{2}+h_{2}\right) t\right]
\end{aligned}
$$

And $P_{d o w n}(t)=1-P_{u p}(t)$

\section{COST ANALYSIS FUNCTION}

The cost analysis function is defined as

$$
G(t)=C_{1} \int_{0}^{t} P_{u p}(t) d t-C_{2} t
$$

Where, $C_{1}=$ revenue cost per unit up time $C_{2}=$ repair cost per unit time

After integration we get,

$$
\begin{aligned}
G(t) & =C_{1}(1+A)\left[\frac{1-\text { exp. }-\left(\lambda+\lambda^{\prime}+e_{1}+h_{1}\right) t}{\lambda+\lambda^{\prime}+e_{1}+h_{1}}\right] \\
& +C_{1} B\left[\frac{1-\text { exp. }-\left(\lambda^{\prime}+\lambda^{\prime \prime}+e_{2}+h_{2}\right) t}{\lambda^{\prime}+\lambda^{\prime \prime}+e_{2}+h_{2}}\right]-C_{2} t
\end{aligned}
$$

Where, $A=\frac{\lambda^{\prime}}{\lambda^{\prime \prime}+e_{2}+h_{2}-\lambda-e_{1}-h_{1}}$ and

$$
B=\frac{\lambda^{\prime}}{\lambda+e_{1}+h_{1}-\lambda^{\prime \prime}-e_{2}-h_{2}}
$$

\section{NUMERICAL COMPUTATION}

\section{Suppose}

$\lambda=h_{1}=0.01, \lambda^{\prime}=h_{2}=0.02, \lambda^{\prime \prime}=0.03, e_{1}=0.04, e_{2}=0.05$ and $C_{1}=2, C_{2}=1$

Putting these values in equations (D), (B), (C) and (A), we get

$$
\begin{aligned}
& \begin{aligned}
A & =\frac{\lambda^{\prime}}{\lambda^{\prime \prime}+e_{2}+h_{2}-\lambda-e_{1}-h_{1}} \\
& =\frac{0.02}{0.03+0.05+0.02-0.01-0.04-0.01}=0.5 \\
B & =\frac{\lambda^{\prime}}{\lambda+e_{1}+h_{1}-\lambda^{\prime \prime}-e_{2}-h_{2}} \\
& =\frac{0.02}{0.01+0.04+0.01-0.03-0.05-0.02}=-0.5
\end{aligned} \\
& P_{u p}(t)=1.5 \exp (-0.08) t-0.5 \exp (-0.12) t \\
& \text { and } G(t)=3\left[\frac{1-\exp (-0.08) t}{0.08}\right]-\left[\frac{1-\exp (-0.12) t}{0.12}\right]-t
\end{aligned}
$$

\section{INTERPRETATION}

Table 3.1 computes the availability of the system decreases as the time increases and Figure 3 shows the graph for the same.

Table 3.2 \& Figure 3.4 shows that cost function increases with time $t$ 
Table 1. Availability function with respect to time

\begin{tabular}{|c|c|c|}
\hline S.No. & t & Pup(t) \\
\hline 1 & $\mathbf{0}$ & $\mathbf{1}$ \\
\hline 2 & 1 & 0.9412143 \\
\hline 3 & 2 & 0.8849018 \\
\hline 4 & 3 & 0.8311036 \\
\hline 5 & 4 & 0.7798319 \\
\hline 6 & 5 & 0.7310743 \\
\hline 7 & 6 & 0.684799 \\
\hline 8 & 7 & 0.6409583 \\
\hline 9 & 8 & 0.5994922 \\
\hline 10 & 9 & 0.5603306 \\
\hline 11 & 10 & 0.5233963 \\
\hline 12 & 11 & 0.4886067 \\
\hline 13 & 12 & 0.4558754 \\
\hline 14 & 13 & 0.425114 \\
\hline 15 & 14 & 0.3962327 \\
\hline 16 & 15 & 0.3691419 \\
\hline
\end{tabular}



Figure 3: Availability as function of time

Table 2. Cost function with respect to time

\begin{tabular}{|c|c|c|}
\hline S.No. & $\mathbf{t}$ & $\mathbf{G}(\mathrm{t})$ \\
\hline 1 & 0 & 0 \\
\hline 2 & 1 & $\mathbf{0 . 9 4 0 8 0 7 3}$ \\
\hline 3 & 2 & 1.7665068 \\
\hline 4 & 3 & 2.4820913 \\
\hline 5 & 4 & 3.092606 \\
\hline 6 & 5 & 3.6030952 \\
\hline 7 & 6 & 4.0185583 \\
\hline 8 & 7 & 4.3439145 \\
\hline 9 & 8 & 4.5839748 \\
\hline 10 & 9 & 4.7434198 \\
\hline 11 & 10 & 4.8267823 \\
\hline 12 & 11 & 4.838435 \\
\hline 13 & 12 & 4.7825814 \\
\hline 14 & 13 & 4.66325 \\
\hline 15 & 14 & 4.4842908 \\
\hline 16 & 15 & 4.2493745 \\
\hline
\end{tabular}



Figure 4 : Cost Profit as function of time 


\section{CONCLUSION}

Table 1 and Figure 3 provide information how availability of the complex engineering repairable system changes with respect to the time when failure rate increases availability of the system decreases.

Table 2 and Figure 4 when revenue cost per unit time $C_{1}$ and $C_{2}$ are fixed, then one can conclude by observing this graph that as cost increases, when time increases.

The further research area is widely open, where one may think of the application of MTTF and sensitivity analysis.

\section{REFERENCES}

[1]. Gupta, P.P.; Agarwal, S.C. : 'Cost function analysis of three state repairable system', Microelectron. Reliab. Vol. 24, pp 51-53 (1984).

[2]. Tyagi, P.K.: 'Cost benefit models of reliability models', Ph.D. Thesis submitted to C.C.S.Univ. Meerut, India (1994).

[3]. Sharma, V., Operational Readiness of a multistage Complex Repairable System.; Bulletin of Pure and Applied Sciences Vol. 31 E P 47-55 (2012) 